Electrocardiography

$15.21 \mathrm{MS} 1$

\title{
INFLUENCE OF SIGNAL FIDELITY ON IMPEDANCE CARDIOGRAPHICALLY- DERIVED VALUES AT RESTING AND ACCELERATED HEART RATES
}

\author{
Barry E. Hurwitz, Liang-Yu Shyu, Chih-Cheng Lu, Sridhar P. Reddy, \\ Neil Schneiderman, and Joachim H. Nagel
}

\author{
Behavioral Medicine Research Center, Departments of Psychology \& \\ Biomedical Engineering, University of Miami, Coral Gables, FL 33124
}

\section{ABSTRACT}

Since the spectrum of the impedance cardiogram (ICG) extends from DC to $50 \mathrm{~Hz}$, any amplifier with an upper band limit less than 50 $\mathrm{Hz}$ can be expected to produce attenuation and distortion of the ICG. This signal attenuation may be systematically enhanced under conditions of high heart rates (HR) when a greater proportion of signal energy will be in the upper frequency range of the ICG spectrum. Therefore, the present study was designed to assess the influence of amplifier bandwidth and signal fidelity on dZ/ttmax, stroke volume (SV), and systolic time intervals (LVET, PEP, QZ, HI). The performance of commonly available commercial systems was tested over a broad range of HRs. The results demonstrated that a digitally differentiated dZ/dt signal using a differentiator with a comer frequency of $50 \mathrm{~Hz}$, when compared with the $15 \mathrm{~Hz}$ comer frequency used in the commercial impedance cardiograph, systematically enhanced the $\mathrm{dZ} / \mathrm{dtm}$ max amplitude and SV measurements as $\mathrm{HR}$ increased. For SV the increase ranged from 17 to $30 \%$ as HR increased from 70 to $150 \mathrm{bpm}$. Moreover, the digitally filtered signal had greater resolution and produced less prolonged PEP and $Q Z$ intervals and greater HI with increasing HR. These findings indicate that impedance cardiographs with insufficient upper bandlimits will differentially influence ICG-derived measurements as $H R$ varies.

\section{INTRODUCTION}

Impedance cardiography is a noninvasive, inexpensive, atraumatic, and relatively unobtrusive lechnique for deriving stroke volume (SV), cardiac output, systolic time intervals and several related cardiovascular parameters. Thus this technique afiords the opportunity to perform a comprehensive cardiovascular functional analysis in the resting and behaving human. The value of impedance cardiography for studying cardiovascular regulation in response to dynamic challenge [1] or for clinical diagnostic assessment [2] depends ultimately upon the signal fidelity of the front-end instrumentation.

\section{SPECTRUM OF THE IMPEDANCE CARDIOGRAM}

To establish the appropriate signal fidelity requirements for the instrumentation used, it is first necessary to determine the ICG spectrum, which has not previously been documented. An impedance cardiograph with a $2 \mathrm{~mA}, 100 \mathrm{kHz}$ constant current source and a wideband $265 \mathrm{~Hz}$ amti-aliasing fitter at the output was used to collect the ICG signal ( $\Delta Z$ ). The ICG spectra of ten continuous cardiac cycles at low (70-89 bpm) and high (130-149 bom) heart rates (HR) were obtained with a spectral resolution of 0.98 $\mathrm{Hz}$. The spectra were then averaged across the 7 subjects at both HR levels. The purpose of this spectral analysis was to specify the bandwidth, noise and dynamic range necessary to provide optimum performance of an ICG amplifier for subsequent event detection and amplitude measurement. Therelore, the upper bandlimit was defined as the frequency where the signal power drops below the power of the noise (i.e., SNA < 1). In Figure 1 (left panel) inspection of the ICG spectrum suggests that the noise component can clearly be Identified as the spectrum baseline. This procedure, however, does not take into account the inherent variation of physiological signals. Since detection algorithms generally are based on the typical appearance of these signals, interindividual variance of signal shape may be considered as a measure of the "physiological noise".
Thus the ICG bandwidth was determined by calculating the average spectra, subtracting the baseline noise and deriving the SNR. The SNR for a given frequency was calculated as the ratio of average power and its standard deviation. Then the upper bandlimit may be determined by locating the frequency where SNR declines below one. This procedure limits the spectral band of the signal to those frequencies where the contribution of the inue signal is larger than that of noise and interferences. Inspection of Figure 1 (right panel) reveals that the ICG spectrum ranges within $30 \mathrm{~Hz}$ at slower HR lovels and increases to $50 \mathrm{~Hz}$ at faster HR levels, when SNR equals one.

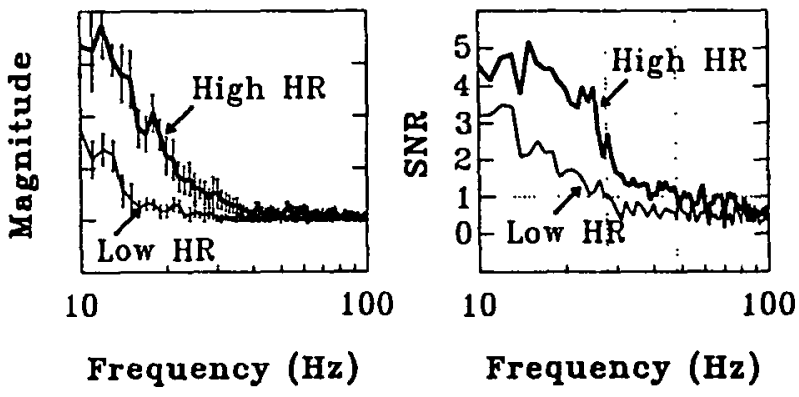

FIGURE 1. Depicted at low (70-89 bpm) and high (130-149 bpm) $H R$ are the mean \pm SD (left) and SNR (right) of the ICG spectum.

In general, the bandwidth of the commercially available impedance cardiographs appears to be insufficient to produce the ICG-dZ/dt whithout attenuation or distortion. Since the Minnesota Impedance Cardiograph (MIC) is the most widely used impedance cardiograph, we have selected it to use in this study. A frequency response analysis of the MIC showed upper band limits of $60 \mathrm{HZ}$ for the $\Delta Z$ channel and $15 \mathrm{HZ}$ for the dZ/dt channel. Since the ICG spectrum extends to $50 \mathrm{~Hz}$, at the very least ICG systems with corner frequencies less than $50 \mathrm{~Hz}$ would be expected to attenuate the signals under conditions of high HRs when a greater proportion of signal energy is in the higher frequency range of the ICG spectrum. Since a HR dependent outcome was anticipated, this study examined the influence of signal fidelity on ICG-derived dZ/dtmax. $\mathrm{SV}$ and systolic time interval indices over a broad range of $\mathrm{HA}$.

\section{METHOD}

Seven healthy men reporting no cardiopulmonary or other medical disorders, aged between 21 and 45 yrs, served as subjects. During the testing session electrocardiogram (ECG), phonocardiogram (PCG) and the ICG signals - $\Delta Z$, dZ/dt and mean thoracic impedance $(\mathrm{ZO})$ - were recorded using a Grass polygraph and the MIC (model 304B). Physiological measurements were oblained in 30-5 samples during rest and during a bicycle exercise procedure in which the workload was adjusted to produce HRs in the following bpm ranges: 70-89, 90-109, 110-129, and 130-149. A standard lead VI ECG configuration was used. The PCG was recorded by placing the phonotransducer (Hewlett Packard 21050A) on the cardiac window in the second intercostal space just left of the sternum. This measure provided confirmation of the location of the ICG-X wave reflecting aortic valve closure. The ICG was derived using a tetrapolar electrode configuration, with bands placed $360^{\circ}$ around the body [ct. 3]. The mean of the front and back distance between 
leads 2 and 3 was measured for later use in calculation of SV using the Kubicek equation [4]. All signals were sampled by an IBM PS/2 Model 70 computer at $1 \mathrm{kHz}$ using an AD converter (DT 2901). Impedance calibration signals for the three ICG measures were also stored in the computer for later conversion of the ICG measurements to the corresponding units.

The ECG, PCG and dZ/dt signals were displayed simultaneously on a computer display on a beat-by-beat basis. A computed $\mathrm{dZ} / \mathrm{dt}$ was also displayed, wherein the derivative of the measured $\Delta Z$ was calculated using a nonrecursive digital differentiator, with a comer frequency of $50 \mathrm{~Hz}$, Iransition bandwidth of $5 \mathrm{~Hz}$ and minimum stopband rejection of $-20 \mathrm{~dB}$. ICG events were located automatically and the displayed event markers could be manually adjusted. The dZ/dtmax was defined as the amplitude difference between B-point and the maximum of the $\mathrm{dZ} / \mathrm{dt}$. Left ventricular ejection time (LVET) was determined as the interval between the $B$ point and the $X$ minimum. The mean Zo during systole for each cardiac cycle and the blood resistivity constant of 135 R.cm were used in the Kubicek equation. Systolic time intervals such as pre-ejection period (PEP), and time to maximal ejection velocity (QZ) as well as the contractility index, Heather index (HI), were also measured. The parameters were scored on a beat-by-beal basis and values were averaged over ten consecutive cardiac cycles within each of the four HR ranges.

\section{RESULTS}

The data analysis used a repeated measures analysis of variance to compare across the four HR intervals the cardiovascular parameters (dZ/dimax, SV, LVET, PEP, QZ and HI) derived from the two measurement methods: the dZ/dt measured from the MIC, with a bandwidth of $15 \mathrm{~Hz}$, and the $\mathrm{dZ} / \mathrm{dt}$ computed from the measured $\Delta Z$ by a digital fitter with a bandwidth of $50 \mathrm{~Hz}$. Table 1 depicts the mean \pm SD of the dZ/dimax and SV for each method during the HR intervals. For both $\mathrm{dZ} / \mathrm{dtmax}$ and $\mathrm{SV}$ signilicant differences between measured and computed methods were observed, with greatest mean differences appearing at the fastest HRs and reducing at the slowest HRs $(p<.005)$. However, at each HR level the $d Z / d t$ max and the $S V$ values derived from computed $d Z / d t$ were greater than values derived from measured dZdt (see Table 1 for $p$ values). For example, the underestimation of SV by the measured $\mathrm{dZ} / \mathrm{dt}$ method ranged from $17 \%$ to greater than $30 \%$ over the HR intervals.

Table 1. Mean ( \pm SD) Ejection Velocity (dZ/dtmax) and Stroke Volume (SV) Comparino Measured dZ/dt and Comouted dZ/dt Heart Rate (bpm)

\begin{tabular}{llllcc}
\hline Parameter & Method & $70-89$ & 90.109 & $110-129$ & $130-149$ \\
\hline dZ/dtmax & measured & $2.0 \pm 0.4$ & $2.9 \pm 0.4$ & $3.0 \pm 0.4$ & $3.1 \pm 0.5$ \\
$(\Omega / s)$ & computed & $2.3 \pm 0.6^{\ddagger}$ & $3.4 \pm 0.4^{*}$ & $3.8 \pm 0.7^{*}$ & $4.1 \pm 0.7^{*}$ \\
SV & measured & $67.3 \pm 27$ & $79.2 \pm 21$ & $74.9 \pm 21$ & $67.5 \pm 16$ \\
$(\mathrm{ml})$ & computed & $78.6 \pm 34^{\infty}$ & $94.4 \pm 28^{\ddagger}$ & $94.8 \pm 30^{\ddagger}$ & $87.9 \pm 23^{\ddagger}$
\end{tabular}

p< .05; ${ }^{\ddagger} p<.005 ; " p<.0005$ ( $p$ values for measured vs. computed)

Figure 2 displays, in a representative subject, greater $\mathrm{dZ} / \mathrm{dtmax}$ obtained using the computed dZ/dt method (thick line) than the dZ/dtmax measured from MIC (thin line); this difference is present at

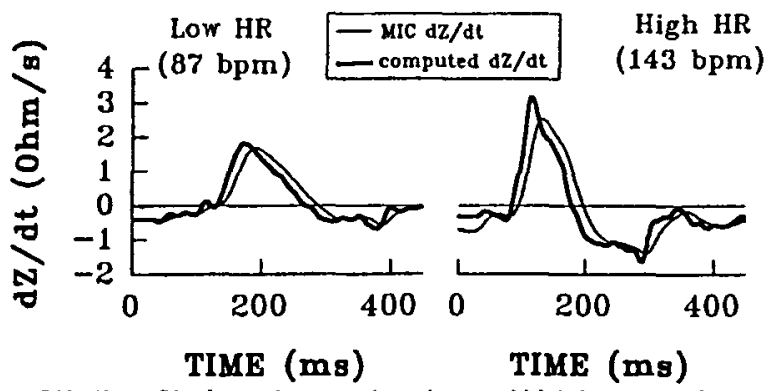

FIGURE 2. Single cycle records at low and high heart rate from a single subject comparing measured MIC dZ/dt with computed dZ/dt. both low (87 bpm) and high (143 bpm) HRs. Note the greater resolution of the relevant signal events ( $B$ point, $d Z / d t$ peak and $X$ wave) in the computed dZ/dt tracings.

Table 2. Mean ( \pm SD) Systolic Time Intervals Comparing Measured dz/dt and comouted dz/dt

Heart Rate (bpm)

\begin{tabular}{lccccc}
\hline Parameter & Method & $70-89$ & $90-109$ & $110-129$ & $130-149$ \\
\hline LVET & measured & $270.5 \pm 16$ & $227.9 \pm 16$ & $208.6 \pm 12$ & $184.4 \pm 15$ \\
(ms) & computed $272.7 \pm 15$ & $227.0 \pm 17$ & $207.4 \pm 13$ & $182.7 \pm 16$ \\
& & & & & \\
PEP & measured & $92.7 \pm 24$ & $59.7 \pm 4$ & $57.8 \pm 3$ & $56.9 \pm 6$ \\
$(\mathrm{~ms})$ & computed & $80.9 \pm 24^{*}$ & $51.3 \pm 4^{*}$ & $48.8 \pm 4^{*}$ & $47.8 \pm 6^{*}$ \\
QZ & measured & $155.5 \pm 22$ & $115.3 \pm 9$ & $108.3 \pm 6$ & $101.7 \pm 6$ \\
$(\mathrm{~ms})$ & computed & $131.6 \pm 22^{*}$ & $91.0 \pm 6^{*}$ & $84.5 \pm 5^{*}$ & $78.5 \pm 3^{*}$ \\
H & measured & $13.6 \pm 5$ & $24.8 \pm 3$ & $27.9 \pm 5$ & $30.8 \pm 6$ \\
$\left(\Omega / s^{2}\right)$ & computed & $18.9 \pm 9^{\infty}$ & $37.5 \pm 5^{*}$ & $45.6 \pm 10^{*}$ & $52.5 \pm 10^{*}$
\end{tabular}

${ }^{\infty} p<.05 ;{ }^{\ddagger} p<.005 ; " p<.0005$ ( $p$ values for measured vs. computed)

Table 2 depicts the effect of the two measurement methods on the systolic time intervals LVET, PEP, QZ and $\mathrm{HI}$. For $\mathrm{HI}$ and $\mathrm{QZ}$ a significant increasing difference between methods was found with increasing HR level ( $p<.0005$ ). However, for PEP decreasing difference between methods was obtained with faster HRs ( $p<$ $.0005)$. Although there was no effect on LVET interval, for PEP, QZ and $\mathrm{HI}$, the computed method resulted in significantly different values than the measured method (see Table 2 for $p$ values).

\section{DISCUSSION}

The primary objective of the present study was to assess the influence of signal fidelity on cardiovascular indices by comparing measurements derived from $\mathrm{dZ} / \mathrm{dt}$ with measurements obtained from the computed derivative of $\Delta Z$ at HRs varying over a broad range. The results showed that the $15 \mathrm{~Hz}$ low pass filter used in the MIC substantially attenuated the dZ/dt amplitude and SV measurements. This effect occurred both at slower HRs and also to a greater extent at faster HRs, wherein greater than $30 \%$ difference in dZdtmax was found. The differential influence ol signal fidelity may, in part, explain the low correlations, reported in a recent validity study, between ICG and nuclear ventriculography SV estimates during exercise when HRs were increased but not during rest [5].

While there was no influence of signal fidelity on LVET, the enhancement of waveform topography when dZ/dt was computed resulted in less latency of the ICG signal events relative to the ECG-Q wave. Consequently, PEP and $Q Z$ intervals were shorter; the larger dZ/dtmax and shorter QZ intervals resulted in greater HI. In summary, these findings indicate that, when an upper bandlimit less than $50 \mathrm{~Hz}$ is used to filter $\mathrm{dZ} / \mathrm{dt}$ an inherent underestimation of dZ/dtmax, SV and $\mathrm{HI}$, and an overestimation of the systolic time interyals, PEP and $\mathrm{QZ}$, occurs. Moreover, the error systematically increases as HR accelerates from resting levels.

\section{REFERENCES}

1. Saab , PG, et al., (1991). J Hypertension. 9:249

2. Fuller, $H$, et al., (1989). Ann Biomed Ena. 17:483

3. Hurwitz, BE et al, (1990). Proc 3rd Annual IEEE Symp ComputerBased Med Systems, IEEE Comouter Soc Press, Wash, p. 228

4. Kubicek, WG, et al., (1966). Aerosoace Med. 37:1208

5. Wilson, MF, et al., (1989). Ann Biomed Eng. 17:475 